\title{
Directed search through autobiographical memory
}

\author{
WILLIAM B. WHITTEN II \\ Bell Laboratories, Holmdel, New Jersey 07733 \\ and \\ JANET MAURIELLO LEONARD \\ State University of New York, Albany, New York 12203
}

\begin{abstract}
Two experiments were designed to study retrieval from a well-ordered set of overlearned information in long-term autobiographical memory. In Experiment 1, each of 161 university students was asked to recall the name of one teacher from each of the 12 preuniversity school years. Recall was cued in forward (Grades 1-12), backward (Grades 12-1), or random order. Backward-ordered search proved most efficient: Fewer students failed to complete the task in that search order, and those who did complete the task were faster than the successful students in the other search orders. Experiment 2 was similar to Experiment 1, except that the 148 students tested were asked to "think aloud" as they attempted recall. From the resulting protocols, 25 categories of verbal reports were identified, including four that reflected retrieval strategies. Omission errors (failures to recall teachers' names) and, with one exception, frequencies of verbal reports decreased as recency increased. Taken together, the results of both experiments show that the recall of one item can indirectly aid the recall of contiguous items and that the probability of recalling an item from autobiographical memory is primarily a function of recency.
\end{abstract}

Who was your third-grade teacher? Such questions tap information that we usually describe as autobiographical. While the term "autobiographical memory" could be used as a synonym for episodic memory (Tulving, 1972), it is more useful to define autobiographical memory as that set of information pertaining to extraexperimentally experienced events. ${ }^{1}$ So defined, the most certain statements that can be made about autobiographical memory are that we know relatively little about its dynamics and that it is of great interest to psychologists and nonpsychologists alike. The reasons we know little about autobiographical memory are obvious: It is difficult to do experiments over very long intervals, and it is even more difficult to exert experimental control over what is, by definition, information stored idiosyncratically throughout one's life. Nevertheless, autobiographical memory is of interest to psychologists who would like to determine the generalizability of typical episodic laboratory phenomena to extraexperimental situations. In addition, this class of memory is of interest to anyone who ever wondered by some events

This report is an extension of a paper presented at the meetings of the Psychonomic Society, Phoenix, November 1979. We wish to thank several people who helped with this project. Stephanie Sorrentino collected the data for Experiment 1. Taren Chaiet and Nancy Downey helped with Experiment 2. Janice Bauhoff helped with a pilot study. Sandra Whitten typed the manuscript. Requests for reprints should be sent to William B. Whitten II, Bell Laboratories, Holmdel, New Jersey 07733. seem to be easily remembered while other events seem hopelessly forgotten.

With few exceptions (e.g., Waldfogel, 1948), most nonclinical studies of autobiographical memory have been reported since 1970 . These recent studies fall into two broad categories. The first category uses Galton's (1907) technique of open-ended association to each of a series of words. On each trial, a single word is presented, and the subject is told to report the first autobiographical episode that the word brings to mind. Five such studies have been published. Testing college students with this procedure, Crovitz and Schiffman (1974) found a linear relation between the $\log$ of report frequency and the $\log$ of report recency such that more reports were from the more recent time periods. Crovitz and QuinaHolland (1976) found that the reports of college students increased as a function of recency over the first 7 years of life. Franklin and Holding (1977) tested subjects whose ages ranged from 25 to 74 years and also found recency effects. McCormack (1979), however, found check-mark-shaped functions when the reminiscences of older people (from 53 to 102 years of age) were plotted against quartiles of the life span. Robinson (1976) tested college students with this paradigm to study word-class effects and found that affect words elicited more recent memories, on the average, than did object or activity words, but that affect-cued events were reported more slowly than were object-cued or activity-cued episodes.

The second category of studies includes a variety of 
techniques used to assess subjects' memories for particular events, such as news items (Botwinick \& Storandt, 1974; Linton, 1975; Sanders \& Warrington, 1971; Squire, 1974; Underwood, 1977; Warrington \& Sanders, 1971; Warrington \& Silberstein, 1970), television shows (Squire, Chace, \& Slater, 1975; Squire \& Slater, 1975; Squire, Slater, \& Chace, 1975), famous race horses (Squire \& Slater, 1975), famous faces (Sanders \& Warrington, 1971; Warrington \& Sanders, 1971), classmates (Bahrick, Bahrick, \& Wittlinger, 1975; Williams, Note 1), teachers (Schonfield, 1969), and all people known personally (Riegel, 1973). Several of these studies have found that memory is better for more recent events (Linton, 1975; Riegel, 1973; Squire, Chace, \& Slater, 1975; Squire \& Slater, 1975; Warrington \& Sanders, 1971; Warrington \& Silberstein, 1970). Underwood (1977) has reported that subjects' accuracy for temporally ordering events in pairs increases as the time between the events increases. Squire, Chace, and Slater (1975) have found the complementary result, that correct temporal ordering of equidistant events in pairs decreases with increasing retention intervals. And Linton (1975), using herself as the sole subject, found both of these results. Some studies have shown differences between recall and recognition performance (e.g., Bahrick et al., 1975; Warrington \& Sanders, 1971; Warrington \& Silberstein, 1970), and one study has attempted to capture the character of extended retrieval by describing recurrent phenomena found in verbal protocols (Williams, Note 1).

Summarizing this previous research, we can say that we now have some information on the frequency of freely recalled memories from the fractions of one's life, the frequency and speed of retrieving events associated with various classes of words, the forgetting functions for recall and recognition over very long time intervals, the ability to order events along the temporal dimension, and some descriptions of the retrieval process as gleaned from self-reports. The type of information missing from the literature thus far is information that would enable a description of retrieval dynamics, information that would allow a prescription for an optimal retrieval strategy, and information that would allow extension of laboratory models of storage-retrieval interactions to the realm of autobiographical memory.

Consider again the question, who was your thirdgrade teacher? The phenomenology of retrieving the answer to such a question is extremely variable. For some people, the answer seems to come automatically, but for other people, the answer is obtained only after a lengthy and obvious memory search. And, of course, some people do not recover the answer at all. What retrieval strategy would be most likely to succeed? Does ordered search along the temporal dimension help? What sorts of information are recovered during a difficult search? How do people define and narrow the search space? The experiments reported here were designed with these questions in mind.

While it is not possible to control for the order or extent of information stored during a person's life, it is possible to manipulate the conditions of retrieval. In order to learn about the relative efficiency of various retrieval strategies, it is only necessary to identify some ordered set of information that is guaranteed to have been stored by all subjects in the experiment. Major news events constitute one possible set of information. Unfortunately, however, we cannot guarantee that all subjects will have experienced all the news events equally or that their knowledge of the events will date from the events' occurrences rather than from later discussions. In addition, it is probable that news events are mostly ignored by young children, so only those events occurring during the adolescent and adult years can be tested. Similar criticisms can be levied against the use of television shows, race horses, sporting events, and so on. In fact, it is very difficult to think of any ordered set of information that is guaranteed to be shared equally by all people. The main source of such shared experience would be a function of a culture's institutions. In the United States, the dominant institution is probably the educational system, and it is true that the vast majority of university students have experienced 12 years of preuniversity schooling. Furthermore, during each of those school years, the majority of students have had one or more teachers with whom daily or semidaily contact occurred. This repeated contact with teachers during the course of a school year surely results in overlearning to the extent that an occasional remembrance of any particular teacher should have relatively small long-term consequences for subsequent retrieval. In addition, it is typical for a student to have had different teachers each year, and for each year to have been punctuated by a 3-month summer vacation. Consequently, for the majority of university students, teachers from these 12 years of school constitute an ordered set of information in memory. Thus, in spite of some minor problems that will be detailed below, teachers from the 12 years of preuniversity schooling constitute a viable set of information for autobiographical memory experiments, since this information is ordered, is overlearned, and is in the experience of most of our subject population. For these reasons, teachers' names were the target memories sought by subjects in our experiments.

\section{EXPERIMENT 1}

The primary question of Experiment 1 was, does the order of memory search affect the speed or likelihood of retrieval from an ordered set of autobiographical memories? A priori, there are reasons to expect that forward-ordered search (Grades 1-12) would be superior, there are other reasons to expect that backward-search (Grades 12-1) would be superior; and there are also reasons to argue that search order would make no difference at all. Consider each of these three positions in turn. Forward-ordered search could be most efficient for three reasons. First, it could be argued that starting 
with first grade provides a primacy advantage. Such an advantage is found in a wide variety of situations ranging from short-term memory (e.g., memory-span tests) to the recall of United States presidents (Roediger \& Crowder, 1976). The primacy items could facilitate recall of a whole set of 12 teachers by serving as an easy-to-find starting point that could guide retrieval of the next item sought. Second, it could be argued that the ordered set of autobiographical information is analogous to a list in serial learning, and it is wellknown that forward recall is much better than backward recall of serially learned lists. (Try to quickly recite the alphabet backward to convince yourself that this is true, even for overlearned sequences.) Third, it can be pointed out that the oldest memories seem less affected by traumatic retrograde amnesia (e.g., Squire, Slater, \& Chace, 1975) and that, of those memories affected by such amnesia, the older ones often return to accessibility first (Barbizet, 1970; Russell \& Nathan, 1946; Talland, 1968). While the implications of these amnesic effects are not entirely clear, it could be postulated that it is most "natural" to recall information in the order in which it was originally stored. This, of course, seems to be one lesson of the serial learning experiments also.

Now consider the argument in favor of backward. ordered search. There are well-known advantages of recency in memory, and these, like primacy effects, occur in long-term, as well as in short-term memory tasks (e.g., Bjork \& Whitten, 1974; Whitten, 1978). If the successful recall of one item from an ordered set facilitates the recall of a contiguous item, then backward-ordered search should be quite efficient.

Finally, consider the arguments for the prediction that search order will have no effects on the speed or accuracy of retrieving the whole set of teachers' names. This prediction is especially interesting, since it can be arrived at under two diametrically opposed conditions. One condition is that the items in the memory set (teachers' names, in this case) exhibit complete independence from each other. In other words, the recall of one item will have no effect on the probability of recalling another item. For example, the successful recall of the name of one's 12th-grade teacher would have no effect on the speed or likelihood of retrieving the name of one's 11th-grade teacher. Success rates and response latencies for recalling the whole set of teachers' names is, therefore, predicted to be the same in conditions requiring forward search, backward search, and randomly ordered search. The other condition under which order of search should not matter is when all the items in the memory set are completely dependent. This means that the successful recall of one item guarantees accessibility to all the items in the set. The notion, then, is one of set activation: All the items in the memory set become accessible simultaneously, and the order of their cued output has no effect on the total recall time or on the probability of complete success. ${ }^{3}$ Of these two extremes, complete independence is possible, although unlikely, since we would expect some teachers to be associated either directly or indirectly. Complete dependence is highly improbable on the basis of the results it predicts: Complete dependence. predicts that a person will either recall all the items in a memory set or will recall none of them. Thus, failure to recall a particular teacher could only be explained by assuming, post hoc, that the teacher was never a part of the set of teachers. This seems unreasonable, since there is no basis to assume, for example, that only your sixth-grade teacher was not integrated into your memory set of "teachers." Given these arguments, it is difficult to maintain the position that search order will have no effects on the retrieval of autobiographical memories. However, it is not at all clear which search order will prove optimal. Experiment 1 was designed to compare the efficiency of forward, backward, and random search orders.

\section{Method}

Subjects. The subjects were 161 introductory psychology students at the State University of New York at Albany who elected to participate in the experiment in order to partially fulfill a course requirement. The mean ages of those students who succeeded in recalling one teacher from each of 12 grades were $18.0,18.0$, and 18.5 years for backward, forward, and random search, respectively. The mean ages of students who failed to complete the recall task were $18.3,18.6$, and 18.6 years, respectively.

Design. Each student was tested in one of three conditions. Each condition required students to recall the name of one teacher from each of 12 years of school. In the forward-search condition, students' recall progressed from the 1 st to the 12 th grade. In the backward-search condition, students' recall began with the 12th grade and worked back to the 1st grade. Each student in the random-search condition was asked to recall the 12 teachers' names in an order that was determined by shuffling the 12 test cards described below.

Each condition was assigned to students until exactly 20 males and 20 females had succeeded in recalling teachers from all 12 grades. For example, as soon as 20 males had successfully recalled 12 teachers in the forward-search condition, no more males were tested in that condition. A running tally of successes was kept so the experimenter would know when to truncate each condition for each sex. Within these constraints, students were assigned to conditions in alternation.

Materials. A deck of 14 computer cards was prepared for each student. The first card of each deck had spaces for the student's name and birth date. On the last card of each deck was printed "The clock time is." A card was printed for each of the first six grades with the phrase "Name of your first [or second, third, fourth, fifth, sixth] grade teacher." Cards for each of the upper six grades read "Name of any of your seventh [or eighth, ninth, tenth, eleventh, twelfth] grade teachers not yet named in this experiment." Card decks for forward-search students had the 12 test cards in ascending order (1-12), decks for backward-search students were arranged in descending order (12-1), and the 12 test cards for each random-search student were shuffled to create a random order.

Procedure. From 1 to 10 students were tested at a time. Each student was seated approximately $2 \mathrm{~m}$ from one of two synchronized digital clocks. The "snooze/second" buttons on these clocks were fastened down so that only the three numerals indicating minutes and seconds were displayed. The bright green numerals were $1.5 \mathrm{~cm}$ high and were reported to be clearly readable by all students. Students did not know what they were going to be asked to recall until they looked through their card decks. In part, the instructions to the students read as follows: 
"We are trying to discover and describe how people remember events from different periods of their lives. The cards in the leck list events which you are to recall. Each of these everts is common to all of us and is in no way embarrassing. The experimental task is very straightforward. We want you simply to recall the event on each card and when you are sure of the answer, write it down in the middle of the card. Please be absolutely certain that each answer is correct before going to the next card, and write only one answer per card. When you reach the last card in the deck, you are to record the time shown on the clock in the front of the room. Then sit quietly until everyone has finished the task. If you cannot recall the event on one of the cards, do not go on to the next card, but continue to try to recall that event until you are successful or until the experimental session is over. The session will last 10 minutes."

The important parts of these instructions were reiterated. Then, as the experimenter told the students to begin, she recorded the time shown by the clocks. (This "start time" was later subtracted from each successful student's "finish time" in order to give the recall latency in seconds.) After the maximum 10 -min recall interval, students were given a questionnaire that asked if they had known that they were going to recall their teachers' names in the experiment. They were then given a feedback sheet that explained the main purpose of the experiment, and they were asked to refrain from telling people about the experiment until the end of the semester.

\section{Results}

The data of a small number of students were discarded for one or more of the following reasons: (1) The student skipped a grade of school. (2) The student had the same teacher for more than 1 year of the first six grades. (3) The student was enrolled in a school system that was outside the United States. (4) The student had been informed about the purpose of the experiment prior to participating. (5) The student recalled teachers from all 12 grades but failed to follow the instruction to list only one name per grade, thereby invalidating the recall latency data. The data from 161 students who did not violate any of these criteria are the basis for all analyses reported below. There are two primary measures of condition difficulty. One is the number of students in each condition who failed to complete the task (failures). The other is the response latencies of those students who did complete the task (successes).

Failure analysis. By design, there were 40 students/ condition who succeeded in recalling 12 teachers. Overall, 41 of the 161 students tested $(25.5 \%)$ failed to complete the task of recalling one teacher's name from each of the 12 grades. The distribution of failures was subjected to a frequency analysis in order to assess the relative difficulty of the three search-order conditions. The relevant data are given in the first column of Table 1. These failure rates show a significant effect of search order $\left[\chi^{2}(2)=10.58, p<.01\right]$. Backward search was clearly the easiest and produced only $9.1 \%$ failures. Forward and random search were not significantly different and produced $29.8 \%$ and $33.3 \%$ failures, respectively.

The second column of Table 1 reports the mean number of teachers recalled by students who failed to recall all 12. Although there are too few data for any formal
Table 1

Number of Failures Preceding $\mathbf{4 0}$ Successes and Mean Number of Teachers' Names Recalled by Failures in Each Search-Order Condition

\begin{tabular}{ccc} 
Search Order & $\begin{array}{c}\text { Number of } \\
\text { Failures }\end{array}$ & $\begin{array}{c}\text { Mean Recall } \\
\text { by Failures }\end{array}$ \\
\hline Backward & 4 & 8.75 \\
Forward & 17 & 5.53 \\
Random & 20 & 8.20 \\
\hline
\end{tabular}

tests, it can be seen that those students who were working backward through memory recalled more teachers than those who were working forward. These are the results expected under the hypothesis that names of the more recent teachers are more accessible. The intermediate performance of the random-search students is also in line with this hypothesis, since random-search students were cued to recall the teachers' names in a mixed order. On the average, for any given number of retrieval requests, the random-search students were cued to recall fewer early-grade teachers than were the forward-search students and were cued to recall fewer later grade teachers than were the backwardsearch students.

Latency analysis. Each successful student's recall latency was calculated by subtracting the experimenterrecorded starting time from the student-recorded finishing time. Table 2 shows the average times required to recall all 12 teachers' names in each condition. Viewing the means (shown in the top row), it appears that backward search was faster than forward search, which was, in turn, faster than random search. There is, however, considerable variability inherent in these sorts of data, and a 3 (conditions) by 2 (sex) analysis of variance showed only a marginal effect of conditions $[F(2,114)=$ 2.26, $\mathrm{MSe}=18,009.07, \mathrm{p}=.109]$ and no evidence for an effect of sex or for a Sex by Conditions interaction $\left(F_{s}<1\right)$. A few students in each condition took exceptionally long to complete the task, and it seems reasonable to assume that these students are probably not representative of their conditions. For example, if an exceptionally slow person in the forward-search condition elected to work backward through memory to gain access to a blocked early-grade teacher, then that student would be mixing forward and backward search and would not be truly representative of the forwardsearch condition. While it is possible that such mixed strategies occurred in each condition, it is less likely that

Table 2

Mean Number of Seconds Required to Recall All 12 Teachers' Names

\begin{tabular}{lccc}
\hline $\begin{array}{c}\text { Subjects per } \\
\text { Condition }\end{array}$ & Backward & Forward & Random \\
\hline All 40 & 180 & 199 & 222 \\
35 Fastest & 157 & 179 & 191 \\
\hline
\end{tabular}


mixed strategies were adopted by the faster students. For these reasons, the latencies of the slowest five students in each condition were deleted and data from the remaining 35 successful students per condition were subjected to a single-factor analysis of variance. The condition means, shown in the bottom row of Table 2 , maintain the same relative ordering and show a significant effect of search order $[F(2,102)=4.09$, MSe $=$ $2,663.50, p=.02]$. Planned comparisons show that backward search was significantly faster than random search $[F(1,102)=7.94, p<.01]$ and marginally faster than forward search $[F(1,102)=3.36, .05<p<.10]$, whereas forward- and random-search times did not differ $(\mathrm{F}<1)$.

\section{Discussion}

The failure analysis and the latency analysis point to the same conclusion: Backward search through autobiographical memory is better than either forward or random search. This result holds several implications for the structure and processes of long-term episodic memory. The most general implication is that we do not have a direct-access or content-addressable memory system for information that is positioned along the temporal dimension. Such a direct-access system would involve complete independence of event memories, so that random retrieval would be as efficient as backwardordered retrieval. In contrast, the results imply that there is some dependence of one memory on another, although the dependence is not complete and may not be based upon direct item-to-item associations. The dependence is not complete, since complete dependence would predict random search to be as successful as backward search whenever at least one teacher was recalled. Additionally, the interdependence of memories is probably not based upon direct associative links of the sort thought to be dominant in serial learning: If the interdependence was based upon such direct associations, we would expect forward search to be better than backward search, just as forward serial recall is better than backward serial recall. Rather, the interdependence probably arises through shared context such that recalling your fourth-grade teacher focuses search processes on context that can lead to contiguous memories, such as those of your third- and fifth-grade teachers. To adopt Norman and Bobrow's (1979) terminology, the description that directs search to fourth-grade memories is more similar to the descriptions of thirdor fifth-grade memories than, for example, to the descriptions of first- or eighth-grade memories. Consequently, the superiority of ordered search over random search can be explained by assuming that ordered search maximizes the use of accessed context and minimizes the need to reaccess context. To explain why backward search is better than forward search, we need only to add the recency principle. In either condition, the context of one retrieved event is assumed to aid retrieval of the contiguous event, but according to the recency principle, the first few events are easier and more likely to be retrieved in backward recall than in forward recall because the first items are more recent in backward than in forward recall. What is missing from this explanation is empirical evidence that, in fact, one's early-grade teachers are less likely to be recalled than one's lategrade teachers. Verification of this assumption was one of several motivations for Experiment 2.

\section{EXPERIMENT 2}

In the first experiment, students who failed to recall a grade's teacher were not allowed to bypass that grade to continue memory search. Because of this procedure, we do not know which grades' teachers are most likely to be recalled, although the number of teachers recalled by failures (see Table 1) hints that the earlier grades are more difficult to access. In Experiment 2; students were once again asked to recall each teacher. However, each student was tested individually, and when he or she told the experimenter that a teacher's name could not be remembered, the experimenter gave permission to skip that grade and continue memory search. Consequently, each subject in Experiment 2 attempted to recall each teacher, even when one or more teacher's names remained inaccessible. This procedure provides the data necessary to determine whether the recency principle or some other serial position function describes longterm au tobiographical memory. ${ }^{4}$

Another motivation for Experiment 2 was to determine what strategies students were employing and what types of information students recalled while trying to access a teacher's name and to determine whether strategies and recalled information vary as a function of grades of school. To obtain this information, each student was tested individually and was asked to "think aloud" while recalling, thereby providing a protocol of strategies used and information retrieved.

Another purpose of Experiment 2 was to check more carefully the accuracy of a student's recall. While it is impractical to attempt verification of each student's recall, it is possible to ask each student to self-verify the list of teachers that was generated during the experiment. The way in which students were asked to do this is described below.

\section{Method}

Subjects. The subjects were 148 introductory psychology students at the State University of New York at Albany who elected to participate in the experiment in order to partially fulfill a course requirement. The mean ages of those students who succeeded in recalling one teacher from each of 12 grades were 19.5, 19.5, and 19.2 years for backward, forward, and random search, respectively. The mean ages for students who failed to complete the recall task were $19.2,19.0$, and 19.9 years, respectively. Students tested in this experiment were slightly older, on the average, than those tested in Experiment 1 because Experiment 1 was completed during the first semester and Experiment 2 was completed during the second semester. 
Design. Each student was tested in one of the three searchorder conditions (forward, backward, or random), as in Experiment 1 . A condition was assigned to students until exactly 10 males and 10 females had succeeded in recalling teachers from all 12 grades in that condition. Within these constraints, students were assigned to conditions in alternation.

Materials. An index card (4 $\times 6$ in.) was printed for each of the first six grades with the phrase "The name of your first [or second, third, fourth, fifth, sixth] grade teacher." The six cards for the upper six grades read "The name of any seventh [or eighth, ninth, tenth, eleventh, twelfth] grade teacher whom you have not named before." The same deck of cards was arranged in forward, backward, or random order and was used for each subject. A blank cover card always topped the deck.

Procedure. Each student was tested individually. The student was seated opposite the experimenter at a table upon which a wooden partition was positioned to prevent the student and the experimenter from viewing each other. A microphone placed on the student's side of the partition was connected to a tape recorder that was placed on the experimenter's side. As in Experiment 1 , the student did not know what he or she was going to be asked to recall until the card deck was read. Instructions to the students were similar to those of Experiment 1, except that students were asked to read each card aloud, to think aloud as they attempted recall, and to speak each answer. Also, there was no clock in the room, and there was no time limit for recall (other than the 30-min experimental session). Students were told to work through the deck as quickly as possible, but to be certain that their answers were correct. The instructions told the students to recall the answer for each card, but, as explained above, students were allowed to skip over a grade if they told the experimenter that they could not remember the teacher. While the student was recalling, the experimenter wrote down the recalled teachers' names. After completing the task, the student was given the list of recalled teachers and was asked to take it home for at least 1 day and to try to verify it in any way possible. Students were counted as successes when they recalled the 12 teachers' names and found no errors during this selfverification period.

\section{Results and Discussion}

Each student's tape-recorded protocol was transcribed, the remarks were categorized by two judges, and the total duration of each recorded protocol was timed. Data were not used if the student had skipped a grade of school, had gone to schools outside the United States, or had been informed about the purpose of the experiment prior to participating. The data of 148 students who did not violate any of these criteria are the basis for all reported analyses.

The failure and latency analyses are reported mainly for completeness, since, as explained below, the procedures of Experiment 2 make these measures relatively unimportant. The results of main interest pertain to error patterns and types, the distribution of selfcorrections among conditions, and the categorization of verbal report information.

Failure analysis. Overall, 88 of the 148 students (59.5\%) failed to recall 1 or more of their 12 teachers. This failure rate is over twice that of Experiment 1, but there are three possible reasons for this. First, students in Experiment 2 were allowed to bypass a grade when retrieval was difficult. Perhaps additional effort, as required in Experiment 1, would have produced success in some of these cases. Second, 33 of the Experiment 2 students who initially recalled all 12 teachers found, upon a day's reflection, that errors had been made. If these students had been counted as successes rather than failures, the overall failure rate of Experiment 2 would have been $37.2 \%$, which is closer to, but still greater than, that of Experiment 1. Third, the verbal reports given by students in Experiment 2 may have been detrimental to recall. It remains to be determined whether vocalization aids or interferes with retrieval of long-term memories.

There were 27,22 , and 39 failures in backward, forward, and random search, respectively. These failure rates were not significantly different $\left[\chi^{2}(2)=5.20\right.$, $.05<p<.10]$. However, if the two ordered-search conditions (backward and forward) are combined and compared with the random condition, the random condition does contain proportionately more failures than would be expected under the null hypothesis $\left[\chi^{2}(1)=4.295, p<.05\right]$. In Experiment 1, failure rates reflected the likelihood of successful retrieval in each condition. In Experiment 2, however, failure rates have a different meaning, since students were allowed to bypass grades whenever retrieval seemed difficult. Thus, the failure rates observed in Experiment 2 are a function of perceived retrieval difficulty as well as actual retrieval probability. Since the relation between perceived difficulty and retrieval probability is not known, interpretations of Experiment 2 failure rates should be tempered. However, it seems reasonable to conclude that random search produces more failures than ordered search under conditions such as those of Experiment 2.

Latency analysis. Because of the "thinking-aloud" requirement of this experiment, the latencies do not hold much importance. Each student's recall time is a joint function of retrieval difficulty and amount of extraneous reportage. Since the latter factor introduces great variability, it is not surprising that an analysis of variance with retrieval order and sex as factors produced no significant results $[F(2,54)=2.31, F(1,54)=1.78$, and $F(2,54)=.45$ for retrieval order, sex, and the interaction, respectively; $\mathrm{MSe}=4,243.41]$.

Error patterns and types. All three search-order conditions exhibited the same general pattern of errors: Students were more likely to forget the names of their early teachers than of their more recent teachers. For clarity, the error data of all three conditions were combined and are shown in Figure 1. Failure to recall a name for a grade's teacher is an omission error, whereas the reporting of a name that was later found to be incorrect (during the self-verification period) is a commission error. As can be seen in Figure 1, omission errors follow a recency function such that errors are less likely for the more recent grades. For example, students who forgot at least one teacher's name were about twice as likely to have forgotten their first-grade teacher's name as they were to have forgotten their sixth-grade teacher's name. Commission errors, on the other hand, were more evenly distributed across grades, such that Grades 14 


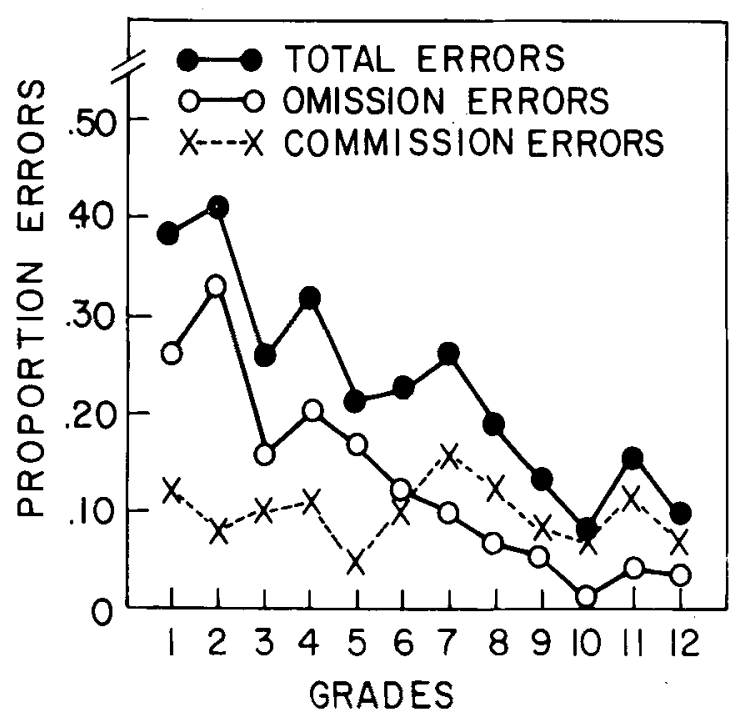

Figure 1. Proportion of total errors, omission errors, and commission errors as a function of grades for the $\mathbf{8 8}$ students who failed to complete the recall task.

averaged $10.5 \%$, Grades 5.8 averaged $10.8 \%$, and Grades 9.12 averaged $8.2 \%$ for the 88 students classified as failures. The reason for this rather even distribution is not apparent, but it may be related to the finding that a majority of commission errors are transposition errors. Students were encouraged, but not required, to explain their commission errors on the self-verification forms. Of 104 commission errors, 55 were explained, and a classification of these is shown in Table 3 . As can be seen, $62 \%$ of these errors are transposition errors in which the student mismatched a teacher with a grade. These were distributed quite evenly with four, two, two, three, two, two, three, five, four, two, three, and two errors reported for Grades 1-12, respectively. The next most frequent type of commission error was the "situation confusion," in which a student retrieved the name of a teacher who was associated with the queried grade of school, but who was not the student's teacher. For example, one student initially reported that Mr. Ricardo had been his 12th-grade teacher, but later he reported that his teacher had been Mr. Sawyer. The student's explanation of this error was that he had wanted Mr. Ricardo to be his 12th-grade teacher, but Mr. Ricardo

Table 3

Types of Commission Errors

\begin{tabular}{lc}
\hline \multicolumn{1}{c}{ Error Type } & Frequency \\
\hline Transposition & 34 \\
Situation Confusion & 9 \\
Acoustic Confusion & 7 \\
Special-Subject Teacher & 2 \\
Image Confusion & 1 \\
Sibling's Teacher & 1 \\
Friend's Teacher & 1 \\
\hline
\end{tabular}

Note-These frequencies are of the 55 errors that students explained. Another 49 errors were not explained by students. had been reassigned to teach 9th grade. In another case of situation confusion, a student initially reported Mr. Meyers as his seventh-grade teacher. Actually, Mr. Meyers had not been the student's seventh-grade teacher, but he did teach seventh grade while the student was in the eighth grade, and during that year, Mr. Meyers dismissed the student from the basketball team. Other types of errors included acoustic confusions, in which the initially reported name sounded like the correct name, and an image confusion, in which the reported teacher appeared similar to the correct teacher. The specialsubjects teacher errors were not full-fledged errors in that these were teachers, but they were not the main elementary school teachers requested by the cue cards. The sibling's teacher and friend's teacher errors are selfexplanatory, although rather unusual sorts of mistakes.

Probably the most important finding shown by the error analysis is that the patterns of omission errors and total errors approximate a simple recency function. There appears to be no primacy advantage, and the probability of correct recall appears to be almost wholly dependent on recency.

Distribution of self-corrections. In the backward-, forward-, and random-search conditions, there were 10,9 , and 14 students, respectively, who recalled 12 teachers during the experimental session, but who then found errors during the self-verification period. These numbers were not significantly different $\left[\chi^{2}(2)=1.27\right]$. Thus, the distribution of commission errors was unaffected by search-order conditions. An important implication of this result is that the differences found among the conditions of Experiment 1 are valid and are not an artifact of undetected commission errors: While some of the students classified as successes in Experiment 1 may have made commission errors, there is no reason to believe that these errors are distributed unequally among the conditions.

Protocol classification. By reading the students' verbal protocols, we were able to identify 25 types of reports. Table 4 gives definitions and representative examples for each report category. Classification of the verbal reports was necessarily subjective, and occasionally, a report could have been classified in more than one way. These cases were rare, however, and each report was classified in only one way, so that the total number of reports tallied would not be inflated. The 25 categories of reports can be clustered so that fewer, higher order categories subsume all the reports. One possible clustering is given in Table 5. The main problem inherent in such higher order clustering is that several of the lower order categories are cross-listed (i.e., the higher order clusters are overlapping). Another problem is that only some of the reports from certain categories fit into particular higher order clusters. Nevertheless, the groupings shown in Table 5 are effective for summarizing, in as few categories as possible, the classes of remarks made by the students during recall. Before discussing these further, it would be useful to observe the frequencies within the 25 individual categories of reports. 
Table 4

Categories, Definitions, and Examples of Verbal Reports

\begin{tabular}{|c|c|c|}
\hline Category & Definition & Example (Cued Grade is in Parentheses) \\
\hline 1. Subject-Enumeration Strategy & $\begin{array}{l}\text { Report of a particular subject } \\
\text { area. }\end{array}$ & $\begin{array}{l}\text { I'm trying to think of what subjects .... Let's see, I } \\
\text { took math. (8) }\end{array}$ \\
\hline 2. Physical Attributes & $\begin{array}{l}\text { Description of appearance, voice } \\
\text { quality, etc. }\end{array}$ & She was a gigantic woman with a scar on her neck. (3) \\
\hline 3. Specific Events & $\begin{array}{l}\text { Report of a particular event that } \\
\text { occurred. }\end{array}$ & I remember her because she gave me a jewelry pin. (1) \\
\hline 4. Locational Search Strategy & $\begin{array}{l}\text { Review of classroom and object } \\
\text { locations. }\end{array}$ & $\begin{array}{l}\text { I'm thinking about where my locker was and where I } \\
\text { used to walk and which classrooms I went into-with } \\
\text { the hopes that I'll see a teacher there. (10) }\end{array}$ \\
\hline 5. Emotional Responses & Expressions of affection, dislike, & Oh, I was in love with her. (2) \\
\hline
\end{tabular}

6. Personality Attributes

7. Life Events of Teachers

fear, etc

Behavioral traits.

Important changes in the teacher's life.

8. Disruptions in Student's Life

Moving, changing schools.

9. Special Titles or Positions

Sister, Dr., Principal, Dean, etc.

10. Special Personal Relationships Neighbor, friend of relative, etc.

11. Directional Search Strategy

Review of grades in order.

\section{Self Corrections}

13. Grade Amnesia

Giving a wrong name, then the correct name.

Report of no memory for the year.

\section{Memory Monitoring}

\section{First Letters}

16. Nicknames

17. Mnemonics

18. First Names

19. Favorite Subjects

20. Performance Evaluations

21. Siblings' Teachers

22. Special Interests of Teachers

\section{Prior Retrieval}

24. Syllables

25. Double Checks

Assessments of memory strength or probability.

Report of the first letter of a teacher's last name.

Any nicknames.

The application of a mnemonic device.

The teacher's first name. Identification of a favorite subject.

Report of a significant grade. Brother's or sister's teacher, also. Teacher's favorite topics, stories, hobbies, etc.

Report of overt or covert retrieval of a teacher before the grade was cued.

Partial recall of the name. Self-questioning the accuracy of a retrieved name.
Sort of the old-fashioned type. (7)

Miss Leathers, who later got married. (3)

It was right in the middle of moving. I really can't remember the teacher at all. (2)

Sister Roseanne. I remember her because she was the Dean of girls. (10)

She was my mother's best friend. (2)

The first was Mrs. Fitch, then I had Mrs. Shelly .... I had Mrs. McCormack. (3)

Mrs. Morello . . . . No, sorry . . . Mrs. Lenahan was my first-grade teacher. (1)

I'm not thinking of anything. I'm drawing an absolute blank. Um . . . junior year .... I am drawing an absolute and entire blank on my junior year. (11)

I can never remember her name for some reason. I tried it before. (2)

Starts with a " $D$ " ... Deacon. (12)

We called her "Barbie Doll" because of the way-because of her appearance. (10)

Mrs. Trecartin. Tree-Car-Tin . . . That's how she told us to remember it. (1)

Lucy, Lucy something ... . Miss Engles. (5)

Mr. Forster, my science teacher. It was my favorite subject. (8)

She gave me my first " $\mathrm{C}$. " (3)

I remember my sister had her before me. (3)

She loved France... and the astronauts. (6)

She came up before under sixth grade. Her name was Mrs. Lugens. (5)

Rabinowitz . . Liebowitz . . something "witz." (2) Third ... Mrs. Gorman ... or Mrs. . . wait .... She was third ... I'm pretty sure it was Mrs. Gorman. (3)'
Verbal report frequencies. Of the 1,024 reports, $66 \%$ were given before a teacher was named or when no teacher was named, and $34 \%$ occurred after teachers were named. Within each of these groups, reports were tallied by grade and condition. In general, there were too few remarks in any particular category to expect differences among either grades or conditions, so the data of all 148 students were combined across searchorder conditions and across the elementary (1-6) and upper (7.12) grades of school. The resulting tallies, along with a composite tally, are given in Table 6 , in which report categories are listed in decreasing order of overall frequency.

The rationale for separately tallying the reports from the first six and last six grades was that a majority of students have one main teacher during each of the first six grades, whereas they have several teachers during each of the last six grades. It was hypothesized that this difference might result in different search strategies and different categories of reported information for the early and late grades. By a sign test on the frequencies of reports per category (the "Both" columns of Table 6), more categories showed more reports for the early grades than for the late grades $(p=.05)$. A look at the actual total frequencies, however, shows that more reports were given for the late grades than for the early grades. This is largely due to a single category of reports, the subject-enumeration strategy category, which was 
Table 5

Higher Order Clusters of Verbal Report Categories

\begin{tabular}{|c|}
\hline \begin{tabular}{l}
\multicolumn{1}{c}{ Strategies } \\
Subject-enumeration strategy \\
Locational search strategy \\
Disruptions in student's life \\
Directional search strategy
\end{tabular} \\
\hline $\begin{array}{l}\quad \text { Imagery } \\
\text { Physical attributes } \\
\text { Locational search strategy } \\
\text { Nicknames (some) }\end{array}$ \\
\hline $\begin{array}{l}\text { Orthography and/or Partial Name Recall } \\
\text { First letters } \\
\text { Syllables } \\
\text { Mnemonics } \\
\text { First names } \\
\text { Nicknames }\end{array}$ \\
\hline $\begin{array}{l}\text { Metamemory and Consequences of Metamemory } \\
\text { Self-corrections } \\
\text { Double checks } \\
\text { Grade amnesia } \\
\text { Memory monitoring } \\
\text { Prior retrieval }\end{array}$ \\
\hline $\begin{array}{l}\quad \text { Emotive Reports } \\
\text { Emotional responses } \\
\text { Special personal relationships } \\
\text { Favorite subjects } \\
\text { Performance evaluations } \\
\text { Specific events (some) } \\
\text { Personality attributes (some) } \\
\text { Nicknames (some) }\end{array}$ \\
\hline $\begin{array}{l}\text { Miscellaneous Specific Information } \\
\text { Life events of teachers } \\
\text { Special titles or positions } \\
\text { Siblings' teachers } \\
\text { Special interests of teachers } \\
\text { Special personal relationships } \\
\text { Specific events }\end{array}$ \\
\hline
\end{tabular}

Note-Individual verbal report categories are listed under the cluster names.

so frequent that it accounted for $34.6 \%$ of all reports and $58.1 \%$ of the late-grade reports. This is most clear in Figure 2, in which report frequencies of all 148 students are summed over categories and are shown for each grade. The function labeled "all reports" shows that more reports were given for the last six grades than for the earlier grades. The curve labeled "subject-enumeration reports" is nearly a step function, with the break in the function occurring between sixth and seventh grades. Finally, and most interestingly, the curve labeled "all other reports" shows that, with the exception of subject enumerations, the number of verbal reports approximates a simple recency function such that less is said about more recent target events. This is exactly the result expected, given the recency function for errors as shown in Figure 1: The earlier the grade, the less likely is successful retrieval (Figure 1), and when retrieval is less likely, more verbal reports are given (Figure 2). These results correspond to common experience, since we only hear people say "I've got it on the tip of my tongue" when retrieval is difficult. When retrieval is easy, there is often no phenomenology of "work" to retrieve and there may be little or no experience of "thinking" to report.

The results given in Figure 2 include reports of all 148 students. Not surprisingly, those students who failed to recall one or more teachers contributed more reports (an average of 7.75) than did students who recalled all 12 teachers (an average of 5.70). However, the distribution of reports across grades is similar for both groups, except that the slope of the recency function is greater for students who failed to complete the recall task.

Retrieval strategies. As clustered in Table 5, we see that surprisingly few retrieval strategies were illuminated by students' verbal reports. Clearly, the dominant strategy involved subject enumeration, which, as pointed out above, was confined almost wholly to the upper six grades. The apparent goal of subject enumeration is to redefine or to select a more specific description of the cued target. The grade number was usually specific enough when one of the first six grades was cued, and the grade number may have been specific enough for nearly $60 \%$ of the upper grades. For the other $40 \%$ of the uppergrade queries, however, it was apparently necessary, or at least easier, to think of a subject than to directly access the name of a teacher.

The next most frequent strategy involved locational search. This method of search corresponds to the classical mnemonic strategy known as the method of loci (Yates, 1966), except that the method of loci usually involves the "artificial" placement of objects or thoughts in particular locations, whereas the locational searches reported in Experiment 2 referred to objects, people, or events that naturally (actually) occurred in the reported locations. The inference can be drawn that the method of loci takes advantage of a search strategy that works for retrieving experienced events and differs from the reported locational searches only in the encoding mode: For the method of loci, objects or events are "mentally experienced" and intentionally encoded at particular locations, whereas the target information of Experiment 2 was actually experienced and, in a sense, incidentally encoded in particular locations.

Reports of disruptions in students' lives are clustered with strategies in Table 5 because in some cases these reports included what might be called "global locational searches." Unlike the specific locational searches described above, the global locational searches involve an active attempt to decide where the students lived, what schools were attended, and so on. Thus, some disruptions served as anchor points for the search and were used to gain a more detailed description of the cued time period.

The remaining identifiable strategy involved directional search. In many instances, this strategy took the form of reviewing those teachers already recalled. It is not intuitively obvious how such a review aids recall, but perhaps the systematic, ordered review helps to retrieve 
Table 6

Verbal Report Frequencies

\begin{tabular}{|c|c|c|c|c|c|c|c|c|c|}
\hline \multirow[b]{3}{*}{ Report Category } & \multicolumn{9}{|c|}{ Time of Report Occurrence } \\
\hline & \multicolumn{3}{|c|}{ Before or Without Recall } & \multicolumn{3}{|c|}{ After Recall } & \multicolumn{3}{|c|}{ Both } \\
\hline & $\begin{array}{c}\text { Grades } \\
1-6 \\
\end{array}$ & $\begin{array}{c}\text { Grades } \\
7-12 \\
\end{array}$ & $\begin{array}{c}\text { All } \\
\text { Grades }\end{array}$ & $\begin{array}{c}\text { Grades } \\
1-6 \\
\end{array}$ & $\begin{array}{c}\text { Grades } \\
7-12 \\
\end{array}$ & $\begin{array}{c}\text { All } \\
\text { Grades }\end{array}$ & $\begin{array}{c}\text { Grades } \\
1-6\end{array}$ & $\begin{array}{c}\text { Grades } \\
7-12 \\
\end{array}$ & $\begin{array}{c}\text { All } \\
\text { Grades }\end{array}$ \\
\hline Subject-Enumeration Strategy & 8 & 206 & 214 & 2 & 138 & 140 & 10 & 344 & 354 \\
\hline Physical Attributes & 63 & 18 & 81 & 23 & 10 & 33 & 86 & 28 & 114 \\
\hline Specific Events & 25 & 7 & 32 & 17 & 25 & 42 & 42 & 32. & 74 \\
\hline Locational Search Strategy & 36 & 32 & 68 & 2 & 1 & 3 & 38 & 33 & 71 \\
\hline Emotional Responses & 20 & 11 & 31 & 17 & 17 & 34 & 37 & 28 & 65 \\
\hline Personality Attributes & 18 & 6 & 24 & 10 & 24 & 34 & 28 & 30 & 58 \\
\hline Life Events of Teachers & 20 & 2 & 22 & 8 & 6 & 14 & 28 & 8 & 36 \\
\hline Disruptions in Student's Life & 20 & 12 & 32 & 0 & 0 & 0 & 20 & 12 & 32 \\
\hline Special Titles or Positions & 23 & 5 & 28 & 2 & 1 & 3 & 25 & 6 & 31 \\
\hline Special Personal Relationships & 7 & 4 & 11 & 6 & 9 & 15 & 13 & 13 & 26 \\
\hline Self-Corrections & 11 & 4 & 15 & 5 & 0 & 5 & 16 & 4 & 20 \\
\hline Grade Amnesia & 12 & 7 & 19 & 0 & 0 & 0 & 12 & 7 & 19 \\
\hline Memory Monitoring & 10 & 6 & 16 & 0 & 1 & 1 & 10 & 7 & 17 \\
\hline First Letters & 9 & 4 & 13 & 0 & 0 & 0 & 9 & 4 & 13 \\
\hline Nicknames & 2 & 7 & 9 & 0 & 2 & 2 & 2 & 9 & 11 \\
\hline Mnemonics & 5 & 2 & 7 & 2 & 1 & 3 & 7 & 3 & 10 \\
\hline First Names & 3 & 5 & 8 & 1 & 0 & 1 & 4 & 5 & 9 \\
\hline Favorite Subjects & 0 & 5 & 5 & 0 & 2 & 2 & 0 & 7 & 7 \\
\hline Performance Evaluations & 2 & 2 & 4 & 1 & 2 & 3 & 3 & 4 & 7 \\
\hline Siblings' Teachers & 2 & 0 & 2 & 4 & 1 & 5 & 6 & 1 & 7 \\
\hline Special Interests of Teachers & 2 & 1 & 3 & 1 & 2 & 3 & 3 & 3 & 6 \\
\hline Prior Retrieval & 4 & 1 & 5 & 0 & 0 & 0 & 4 & 1 & 5 \\
\hline Syllables & 4 & 1 & 5 & 0 & 0 & 0 & 4 & 1 & 5 \\
\hline Double Checks & 0 & 0 & 0 & 3 & 0 & 3 & 3 & 0 & 3 \\
\hline
\end{tabular}

the cognitive context within which the searched-for item is located. In any case, directional search was a relatively rare strategy that occurred in approximately $1 \%$ of the grades in backward and forward searches and $2 \%$ of the grades in random search. One implication of the rarity of this strategy is that the nominal search orders are, for the most part, the functional search orders. That is, there is no reason to doubt that almost all of the searches in Experiments 1 and 2 were performed in the cued order.

Imagery. Many of the 25 categories of reports may involve imagery, but the cluster of imagery-related reports listed in Table 5 is conservative and lists those reports that most clearly do. Of these, physical attributes were most commonly reported and, in fact, were the second most frequent of all types of reports. This underscores the widely known intimacy between the names of people or objects and their appearances. The retrieval of the name often evokes the image and vice versa. Other reports that reflect imagery include the locational search strategy and some nicknames, such as the example nickname given in Table 4.

Orthography and/or partial name recall. Orthographic attributes, including first letters, syllables, and possibly mnemonics, were rarely reported: They made up only $2.7 \%$ of the remarks and occurred in only $1.6 \%$ of the trials. This contrasts with Brown and McNeil's (1966) tip-of-the-tongue results, which give the impression that word fragments and characteristics are fairly often accessible before retrieval is complete: Their subjects produced such reports on $13.1 \%$ of their trials. On the other hand, Brown and McNeil do not report much evidence for imagery preceding recall, whereas such reports are common in our data. These differences are probably traceable to the different procedures used in their experiment and in ours. Brown and McNeil specifically asked their subjects to report the sorts of attributes that may be labeled orthographic, and that is the category of partial recall information that was obtained. We did not constrain or bias our students toward any category of report, and our results show that many categories of attributes, especially imagery, predominate over orthographic features.

In addition to orthographic attributes of a teacher's last name, recall of the first name or nickname may be classified as partial name recall. In these instances, the students have accessed the correct teachers but have produced names other than those cued. Since 17 of the 20 first names and nicknames were reported before the teachers' proper names, it is probable that these were the dominant labels given to those teachers by the students.

Metamemory and consequences of metamemory. Metamemory is usually defined as a person's knowledge of his memory (Flavell, 1971). Such knowledge can be subdivided into general knowledge about the limitations, strategies, abilities, and so on, of one's memory and 


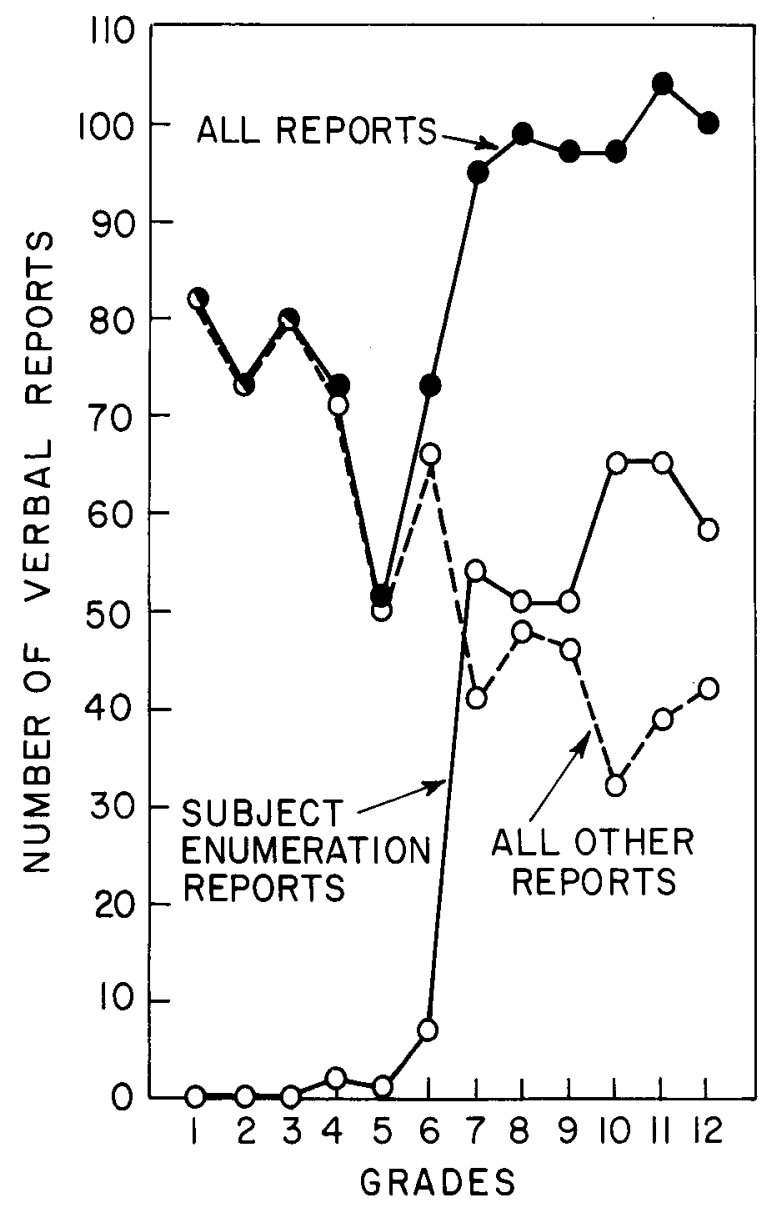

Figure 2. Frequencies of all categories of verbal reports, subject-enumeration reports, and all reports except subject enumerations, shown as a function of school grades.

specific assessments of ongoing memory processes, retrieval attempts, and so on (Wellman, 1977). Table 5 shows a cluster of five report categories that either can be classified as metamemory comments or can be considered to be the consequences of metamemory. Grade amnesia and memory monitoring both fit the definition of the second subdivision of metamemory as defined above. These two report categories could be combined under the "memory monitoring" heading, but they have been separated to emphasize the different focus of the reports in the two categories. Those reports classified as memory monitoring were specific remarks about the cued grade or teacher, whereas the reports classified as grade amnesia were global statements about the whole year or grade. We do not believe that grade amnesia would have persisted if these students had been given additional retrieval cues. However, when subjects were given the cue to recall a teacher, there were 19 instances in which they claimed to remember nothing at all about the grade or year. It should be noted that these cases are probably not identifiable with "childhood amnesia," since they did not extend over a severalyear period and they were not confined to the early grades.
Two other report categories that could be combined are the "self-corrections" and the "double checks." The distinction between these two categories is not in the type of process engaged, but rather in the outcome of the additional processing. For self-corrections, students questioned the validity of a retrieved name, decided it was incorrect, and tried to retrieve the correct name. For double checks, students questioned the validity of a retrieved name and decided that the name was correct. Thus, both report categories could be classified as "verifications" of the sort described by Williams (Note 1).

Reports of prior retrieval are consequences of metamemory in the sense that such reports are possible only when students are aware of previous covert or overt retrievals. Such awareness is a form of memory monitoring in which what is monitored is the updated strength or accessibility of a teacher's name.

Emotive reports. It is difficult to clearly cluster all those report categories that convey emotive information. Certainly, the emotional responses, special personal relationships, favorite subjects, and performance evaluations categories should be included in this cluster. Some, but not all, of the reports from the other categories listed in Table 5 seem to include emotive emphasis. The central question about this cluster of reports is, how does it affect retrieval? In contrast with each of the clusters discussed above, the remarks in this category were about as likely to follow the recall of a teacher's name as to precede it. Thus, it may be the case that many of these reports are based upon incidentally retrieved information that is tightly associated with the name. Such reports could be a consequence of retrieval rather than a precursor of retrieval. On the other hand, almost half of the emotive reports preceded retrieval of the teacher's name and may have facilitated the name's recall. For example, it is easy to imagine that students can focus on the general context of the grade, conjure up an image of the teacher, retrieve facts about favorite activities, personality attributes, or specific events, and then retrieve the teacher's name. In such cases, the products of pre-name retrieval may serve as context for the specific name search.

Miscellaneous specific information. This is admittedly a catchall cluster containing a wide variety of reports about specific events, interests, and so on. As for the emotive cluster, reports from this cluster were about as likely to be given after the teacher's name was recalled as before. Also, as for the reports in the emotive cluster, the reports in this cluster may be consequences of name retrieval in some cases and may precede and serve as context for name retrieval in other cases.

\section{GENERAL DISCUSSION}

We now have a prescription for an optimal retrieval strategy, a description of retrieval dynamics, and some evidence as to which features of laboratory models apply to autobiographical memory. Experiment 1 was 
designed to provide quantitative information about the optimal order in which to search through autobiographical memory. The failure rates and the retrieval latencies of successful students in the three search-order conditions show clearly that backward-ordered search is most efficient. It was reasoned earlier that random search could be as efficient as ordered search if items in the memory set were stored in either a completely dependent or completely independent fashion. Since a form of ordered search was superior, these possibilities are eliminated. There were good reasons to expect each of the ordered-search conditions to be superior. The reasons given in support of forward-ordered search are discredited for long-term search of the sort explored by our experiments. Specifically, there is no primacy advantage, the sequence of items is apparently not stored in a form analogous to serially learned materials, and information is not necessarily recalled more easily in the order of its original storage.

The description of the retrieval dynamics that make backward search optimal assumes two principles: that the context of one retrieved item facilitates the retrieval of a contiguous item and that recent information is more accessible than remote information. The importance of context was implicated by the finding that ordered search was better than random search. As Figure 1 clearly shows, the recency assumption was confirmed by Experiment 2.

The recency function exhibited in Experiment 2 is in agreement with recency functions reported by Crovitz and Quina-Holland (1976), Crovitz and Schiffman (1974), Franklin and Holding (1977), Linton (1975), Riegel (1973), Squire, Chace, and Slater (1975), Squire and Slater (1975), Warrington and Sanders (1971), and Warrington and Silberstein (1970). Obviously, the recency function is not a novel or surprising result. A new result provided by Experiment 2 is that retrieval of information from a well-ordered set contains no primacy advantage. None of the previously reported studies tested memory for a well-ordered set of information that had a defined first item. Experiment 2 did have a defined beginning item, yet no primacy effect was observed. This result has implications for the generalizability of laboratory models to autobiographical memory. In general, the behaviors exhibited in the present experiments match well-known laboratory findings. For example, the importance of context during memory search is a familiar idea that is basic to many models of retrieval. However, the absence of a primacy effect in the present data limits the analogies that may be drawn between these results and typical laboratory results. Specifically, our results seem to parallel the simple list-recency function in which no primacy advantage is found (e.g., Bjork \& Whitten, 1974, Experiments 2 and 3 ), rather than the more commonly studied bow-shaped functions found for recall of single, discrete lists. An implication of these results is that primacy alone may not be a sufficient condition for superior access in memory. A prerequisite for the occurrence of primacy effects may be the encoding of a sequence of information as an interrelated set. Even though the teachers' names in our experiments constitute a semantic category, and even though they are an ordered set with defined endpoints, it is improbable that they reside in memory as an organized, interrelated set.

In our experiments, the accuracy of recall was not verified with school records. While it is possible in principle, it is not feasible in actuality to gain access to the records of over 300 students. However, two results of the error analysis show that it is reasonable to accept our findings as an accurate reflection of memory. One result is that the commission errors of Experiment 2 were found to be distributed equally among search-order conditions. Thus, it is unlikely that such errors contributed to differences among search-order conditions found in Experiment 1. The second result is that all three search orders produced recall recency functions in Experiment 2. If students were more biased to fabricate answers in some search orders than in others, we would not expect to find the same recency functions in all conditions. Since similar functions were found, it is unlikely that such biases were a factor in these experiments.

Experiment 2 complemented Experiment 1 by showing a recency function for teachers' name recall and by showing the equal distribution of commission errors. Experiment 2 went beyond these purposes, however, and provided us with considerable qualitative information about the strategies used and the information retrieved while searching through autobiographical memory. In all, 25 verbal report categories were identified. Although any of these report categories may have resulted from some sort of search strategy, there were only four report categories that clearly should be labeled "strategies." While the verbal content of these strategy reports is specific to the task demands of our experiment, the argument can be made that each of these four categories represents a generally useful strategy with which to tackle memory search. Whereas the subject-enumeration strategy is obviously specific to our target information, it represents the general heuristic of narrowing the search space by redefining it in terms of a subset of possible answers. The locational search strategy was useful to some students we tested, but it also should be useful in other situations in which the target memory contains spatial-locational information. The disruptions in students' lives were recalled in relation to the searched-for years of school in our experiment, but similar disruptions should be useful for narrowing and limiting the search space in many tasks. (These disruptions need not be the large ones, such as moving or changing schools, as found in our experiment; they could be any sort of cognitive landmark that segments the flow of information.) And finally, the directional search strategy we found represents the general strategy of reinstating the original temporal 
ordering of a series of events, thereby gaining access to memories that lie somewhere within the reinstated temporal series. Thus, four general strategies have been identified and could be given the more general names of target-subset selection, imagined-location traversal, cognitive landmark identification, and temporal ordering, respectively.

In addition to search strategies, the categories of verbal reports revealed a wide variety of information recalled during search. The rank ordering of these reports by frequency of occurrence can be considered to be an index of relative importance. This index, in turn, should be useful in guiding selection of the more important memory attributes for further study. Similarly, the clustering of these report categories, as in Table 5, should help focus attention on the more important aspects of memory search.

Retrieval protocols have been analyzed in one previously reported study of autobiographical memory. Williams (Note 1) had four subjects "think aloud" while recalling as many high school classmates as possible. His study differed from ours in that recall was open-ended and lasted several hours, the to-be-recalled information was not well-ordered and was not necessarily overlearned, and search order was not directed. In spite of these differences, there are several similarities between his protocols and ours. Williams reported partial name recalls, self-corrections, and incidental recall of a variety of specific information, as did we. He also found fabrications (commission errors), attempts to verify retrieved names (similar to our double checks), and establishment of search contexts (similar to some of our strategies). Williams also found that his subjects got involved in extended retrieval attempts, made inferences to fill in partial recalls, and were sometimes distracted from retrieval by the recall of similar but incorrect information. None of these phenomena were of much importance in our more defined, content-limited retrieval situation. Five strategies were reported by Williams. "Activity search" corresponds to our subject-enumeration strategy in that it is a form of target-subset selection. "Location scanning" and "image scanning" correspond to our imagined-location traversal. "General association" (free association from one context to another) and "name generation" (systematic generation of common names) had no analogues in our experiment and are probably attributable to the particular task demands of Williams' study.

\section{CONCLUSIONS}

Backward-ordered search through long-term autobiographical memory is more efficient than either forward-ordered search or random-access search. This conclusion is based upon two independent results of Experiment 1: (1) Significantly fewer students failed to complete the retrieval task in the backward-search condition than in either of the other search conditions.
(2) The students who succeeded in completing the task took less time to retrieve in the backward-search condition than in either of the other search conditions. These results are explained by assuming that the context of an item retrieved from a well-ordered series aids the retrieval of a contiguous item more than it aids the retrieval of a noncontiguous item and by assuming that recent items are easier to retrieve than are remote items. Experiment 2 confirmed the second assumption, since the accessibility of teachers' names was found to increase with recency. Experiment 2 also showed that the frequency of verbal reports, in general, is an inverse function of recency. Another way to say this is that the frequency of verbal reports is a function of retrieval difficulty such that students generate more verbal reports for the more difficult retrieval targets when asked to "think aloud." In our data, the one exception to this generalization was that the number of subject-enumeration strategy reports was greater for the six most recent grades. If we had specified one particular teacher for each of the upper grades, this strategy probably would have been unnecessary, and the remaining verbal reports probably would have been a function of recency.

The verbal reports allowed the identification of 25 categories of retrieved information, and the frequencies within these categories provided a qualitative estimate of attribute importance. Some of the reports reflected search strategies, and four general strategies for memory search were identified. These were target-subset selection, imagined-location traversal, cognitive landmark identification, and temporal ordering.

\section{REFERENCE NOTE}

1. Williams, M. D. The process of retrieval from very long term memory (Tech. Rep. 75). La Jolla, Calif: University of California, San Diego, 1978.

\section{REFERENCES}

Bahrick, H. P., Bahrick, P. O., \& Wittlinger, R. P. Fifty years of memory for names and faces: A cross-sectional approach. Journal of Experimental Psychology: General, 1975, 104, 54-75.

Barbizet, J. Human memory and its pathology. San Francisco: Freeman, 1970.

Bjonk, R. A., \& WhitTen, W. B. Recency-sensitive retrieval processes in long-term free recall. Cognitive Psychology, 1974, 6, 173-189.

Botwinick, J., \& Storandt, M. Memory, related functions and age. Springfield, Ill: Thomas, 1974.

Brown, R., \& McNeill, D. The "tip of the tongue" phenomenon. Journal of Verbal Learning and Verbal Behavior, 1966, 5, .325-337.

Crovitz, H. F, \& Quina-Holland, K. Proportion of episodic memories from early childhood by years of age. Bulletin of the Psychonomic Society, 1976, 7, 61-62.

Crovitz, H. F., \& Schiffman, H. Frequency of episodic memories as a function of their age. Bulletin of the Psychonomic Society, 1974, 4, 517-518.

Flavell, J. H. First discussant's comments: What is memory 
development the development of? Human Development, 1971, 14, 272-278.

Franklin, H. C., \& Holding, D. H. Personal memories at different ages. Quarterly Journal of Experimental Psychology, $1977,29,527-532$.

Galton, F. Inquiries into human faculty and its development. New York: Dutton, 1907.

Linton, M. Memory for real-world events. In D. A. Norman \& D. E. Rumelhart (Eds.), Explorations in cognition. San Francisco: Freeman, 1975.

McCormack, P. D. Autobiographical memory in the aged. Canadian Journal of Psychology, 1979, 33, 118-124.

Norman, D. A., \& Boвrow, D. G. Descriptions: An intermediate stage in memory retrieval. Cognitive Psychology, 1979, 11 107-123.

RIEGEL, K. F. The recall of historical events. Behavioral Science, 1973, 18, 354-363.

Roвinson, J. A. Sampling autobiographical memory. Cognitive Psychology, 1976, 8, 578-595.

Roediger, H. L., III, \& Crowder, R. G. A serial position effect in recall of United States presidents. Bulletin of the Psychonomic Society, 1976, 8, 275-278.

Russell, W. R., \& Nathan, P. W. Traumatic amnesia. Brain, $1946,69,280-300$.

Sanders, H. I., \& Warrington, E. K. Memory for remote events in amnesic patients. Brain, 1971, 94, 661-668.

SCHONFIELD, D. In search of early memories. VIIIth International Congress of Gerontology. Washington, D.C: International Association of Gerontology, 1969.

Squire, L. R. Remote memory as affected by aging. Neuropsychologica, 1974, 12, 429-435.

Squire, L. R., Chace, P. M., \& Slater, P. C. Assessment of memory for remote events. Psychological Reports, 1975, 37, 223-234.

Squire, L. R., \& Slater, P. C. Forgetting in very long-term memory as assessed by an improved questionnaire technique. Journal of Experimental Psychology: Human Learning and Memory, 1975, 1, 50-54.

Squire, L. R., Slater, P. C., \& Chace, P. M. Retrograde amnesia: Temporal gradient in very long term memory following electroconvulsive therapy. Science, 1975, 187, 77-79.

Talland, G. A. Disorders of memory and learning. Baltimore, Md: Penguin, 1968.

Tulving, E. Episodic and semantic memory. In E. Tulving \& W. Donaldson (Eds.), Organization of memory. New York: Academic Press, 1972.

UNDERWOOD, B. J. Temporal codes for memories: Issues and problems. Hillsdale, N.J: Erlbaum, 1977.

WALDFogen, $S$. The frequency and affective character of childhood memories. Psychological Monographs, 1948, 62, Whole No. 291.

Warrington, E. K., \& Sanders, H. The fate of old memories. Quarterly Journal of Experimental Psychology, 1971, 23, 432-442.
Warrington, E. K., \& Silberstein, M. A questionnaire technique for investigating very long term memory. Quarterly Journal of Experimental Psychology, 1970, 22, 508-512.

Wellman, H. M. Tip of the tongue and feeling of knowing experiences: A developmental study of memory monitoring. Child Development, 1977, 48, 13-21.

Whitten, W. B., II. Output interference and long-term serial position effects. Journal of Experimental Psychology: Human Learning and Memory, 1978, 4, 685-692.

Whitty, C. W. M., \& Zangwill, O. L. Traumatic amnesia. In C. W. M. Whitty \& O. L. Zangwill (Eds.), Amnesia. London: Butterworths, 1966.

Williams, M., \& Zangwill, O. L. Memory defects after head injury. Journal of Neurology, Neurosurgery, and Psychiatry, 1952, 15, 54-58.

YATES, F. A. The art of memory. Chicago: University of Chicago Press, 1966.

\section{NOTES}

1. Autobiographical memory, by this definition, includes all such events, whether experienced "directly," via mass media, or however. This definition is at variance with some writers (e.g., McCormack, 1979; Robinson, 1976). Our definition includes what others have called "memory for real-world events" or "memory for naturally occurring events."

2. We do not want to give the impression that all amnesias follow a temporally dependent pattern. Recovery from retrograde amnesia may include "islands" of memory that are not temporally ordered (Witty \& Zangwill, 1966), and patients who have recovered normal memory functions following minor accidents may have residual memory defects, or memory gaps (Williams \& Zangwill, 1952).

3. Recall latency differences among search-order conditions could arise if initial access to the memory set is faster via some serial positions than via others. Also, output times for the remaining items could be affected if the required order of output differs from a memory set's directional interassociations. However, "complete dependence," as it is used here, assumes equal associations among all items and would predict that the time necessary to recall the second through the last items in the memory set would be equal for all search-order conditions. In any case, complete dependence predicts no differences in success rates across conditions.

4. The astute reader may wonder whether a recency function would be affected by the fact that most students have one main teacher for each of the first six grades but have several teachers for each of the last six grades. This fact alone should not obscure an overall recency trend, but it may affect a change in the slope between the halves of the function.

(Received for publication November 10, 1980; revision accepted April 23, 1981.) 